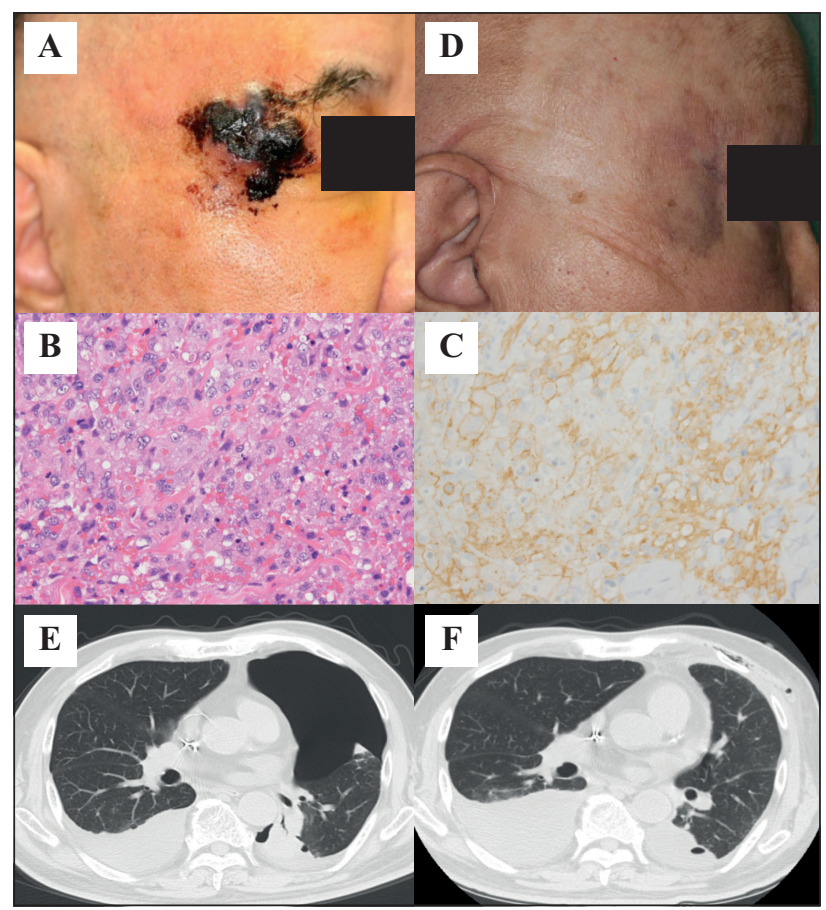

Figure 1. A) Irregular and ill-defined purpura with a bloody crust on the right side of the forehead. B) Dense dermal proliferation of large, round, atypical endothelial cells with atypical mitoses (hematoxylin-eosin staining; original magnification: $\times 200$ ). C) Immunohistological staining for CD31 (original magnification: $\times 200$ ). D) The tumour mostly disappeared two months after the initial treatment. E) Chest computed tomography before the intrapleural paclitaxel treatment shows left-sided pneumothorax and bilateral pleural effusion; F) marked improvement of the left-sided pneumohemothorax by the intrapleural administration of paclitaxel and pleurodesis using OK-432.

for the left side, and was successfully treated in the same manner as for the left-sided lesion. Mild chest pain was the only adverse event. The metastasis of bilateral lung parenchyma rapidly exacerbated six months after the treatment of left-sided pneumohemothorax, and the patient died of the disease.

Intrapleural administration of a taxane preparation for pleural effusion due to malignant tumours has been reported [1-4]. Perng demonstrated the efficacy of this procedure in 14 patients with non-small-cell lung cancer (NSCLC) [1]. After a four-week observation period, the overall response rate of pleural effusion was $92.9 \%$, with a complete response rate of $28.6 \%$. Docetaxel was also beneficial for controlling malignant pleural effusion in NSCLC and breast cancer patients [2]. Adverse events associated with the intrapleural administration of a taxane preparation include chest and/or shoulder pain, fever, nausea, and an increase in transaminases. Renal failure and infection are relatively rare, but occasionally very severe [1,2]. Our patient is the first case of angiosarcoma-related pneumohemothorax treated with the intrapleural administration of a taxane preparation. The prognosis of angiosarcoma is very poor, particularly with pneumohemothorax. The intrapleural administration of a taxane preparation is not a radical cure, but may be used as palliative care. Our patient spent

his last few months with his family at home because the pleural effusion was controlled by the intrapleural administration of paclitaxel. It is difficult to estimate the impact of intrapleural administration of paclitaxel on prognosis. However, the intrapleural administration of a taxane preparation is an option for palliative care for angiosarcoma patients with pneumohemothorax.

Disclosure. Financial support: none. Conflict of interest: none.

${ }^{1}$ Department of Dermatology,
${ }^{2}$ Department of Thoracic,
Cardiovascular and General Surgery,
Faculty of Medicine, Institute of
Medical, Pharmaceutical and Health
Sciences, Kanazawa University,
Kanazawa, Japan
<yasuhito@med.kanazawa-u.ac.jp>

1. Perng RP, Chen YM, Wu MF, et al. Phase II trial of intrapleural paclitaxel injection for non-small-cell lung cancer patients with malignant pleural effusions. Respir Med 1998; 92: 473-9.

2. Jones DR, Taylor MD, Petroni GR, et al. Phase I trial of intrapleural docetaxel administered through an implantable catheter in subjects with a malignant pleural effusion. J Thorac Oncol 2010; 5: 75-81.

3. Wang $X$, Zhou J, Wang $Y$, et al. A phase I clinical and pharmacokinetic study of paclitaxel liposome infused in non-small cell lung cancer patients with malignant pleural effusions. Eur J Cancer 2010; 46: 1474-80.

4. Lombardi G, Nicoletto $M O$, Gusella $M$, et al. Intrapleural paclitaxel for malignant pleural effusion from ovarian and breast cancer: a phase II study with pharmacokinetic analysis. Cancer Chemother Pharmacol 2012; 69: 781-7.

doi: $10.1684 /$ ejd.2018.3318

\section{Candidal vulvitis: a possible trigger for lichen striatus}

A 33-year-old woman presented with a pruritic rash on her left leg. Dermatological examination revealed an erythematous, mildly oedematous vulva and small, flat, erythematous, blaschkoid papules extending from the left inguinal fold to the medial side of the left knee (figure 1A, B). Approximately one month prior, she had visited a gynaecology clinic due to an itchy vulva, and received a corticosteroid cream for suspected allergic vulvitis. The pruritus persisted after one week of treatment, and ornidazole at $500 \mathrm{mg} / \mathrm{d}$, ciprofloxacin at $1 \mathrm{~g} / \mathrm{d}$, and a daily pessary combining $500 \mathrm{mg}$ of metronidazole and $100 \mathrm{mg}$ of miconazole nitrate were prescribed for "vulvovaginitis". Her symptoms slightly improved, however, a blaschkoid rash appeared three weeks later on her left leg. At the time of her first visit to our clinic, the patient's complete blood count, erythrocyte sedimentation rate (ESR), and CRP were within normal range. Skin swabs were taken in order to identify a possible pathogen. Sheep blood agar 


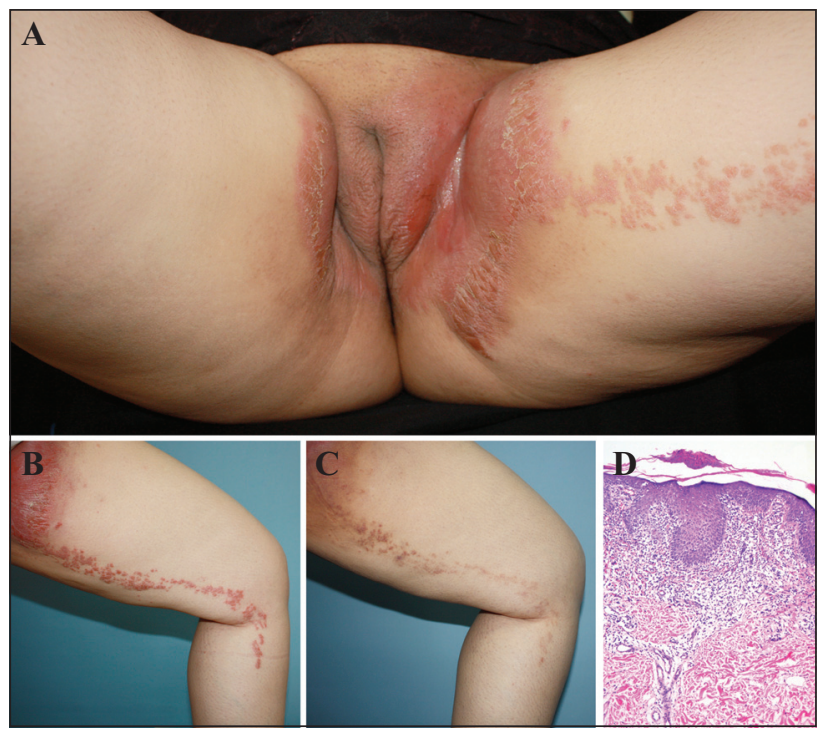

Figure 1. A) A 33-year-old patient with an erythematous and mildly oedematous vulva without apparent abscess formation or suppuration, as well as flat, grouped erythematous papules and plaques extending distally from an erythematous, macerated left inguinal fold. B) Erythematous flat papules and plaques extending in a blaschkolinear pattern along the medial border of the left thigh. C) Three months after topical pimecrolimus and clotrimazole treatment, the blaschkolinear inflammation almost completely regressed, except for a group of papules near the inguinal fold; mild vulvar and inguinal hyperpigmentation can be seen. D) Focal epidermal parakeratosis, psoriasiform acanthosis, liquefaction degeneration in epidermal basal layer, and dermal band-like lymphocytic infiltration $(\mathrm{H} \& \mathrm{E} ; \times 100)$.

cultures were negative, but the potassium hydroxide $(\mathrm{KOH})$ test revealed pseudohyphae and budding yeast cells, consistent with candidal infection. Skin biopsy of the blaschkoid papules was performed. Clotrimazole cream for inguinal intertrigo and pimecrolimus cream for the blaschkolinear inflammation, both twice daily, were started, and remission of vulvitis was observed after ten days. Skin biopsy showed focal parakeratosis, irregular acanthosis, and liquefaction degeneration in the epidermal basal layer and a dermal, band-like lymphocytic infiltration, suggestive of lichen striatus (LS) (figure 1D). The patient continued with daily application of pimecrolimus cream. Twelve weeks later, the blaschkolinear inflammation had nearly completely regressed, except for a group of papules near the inguinal fold (figure 1C).

LS is an uncommon, acquired, self-limiting dermatosis of unknown aetiology, which is frequently observed in children and typically manifests as unilateral, erythematous, asymptomatic, flat papules and/or plaques along Blaschko's lines. Some authors differentiate LS from similar lesions observed in adults by classifying the latter as blaschkitis, whereas others consider such a classification to be unnecessary [1]. Although viral infections, trauma, vaccines, and various drugs have been reported as possible triggering factors, the exact aetiopathogenesis of this condition remains unclear [2]. Clinical and histopathological features of LS suggest a T-lymphocyte-mediated immune reaction against some keratinocytes that have likely acquired antigenic properties due to underlying genetic mosaicism and molecular mimicry [3]. Reports of familial cases appear to contradict this hypothesis [4-6]. Happle proposed a retrotransposon theory to explain such blaschkoid dermatoses [7]. Transposable elements (TE), also called 'jumping genes', are DNA sequences that can move to different sites in the genome and affect expression of nearby genes. Members of the retrotransposon subgroup do not move on their own but require reverse transcription to form a TE. It is possible that retrotransposon-induced alteration of gene expression of a keratinocyte clone may alter the clone's antigenic properties and lead to blaschkolinear inflammation [6, 7]. In some cases, a histopathological phenomenon known as "syringotropism", which refers to lymphocytic infiltration of eccrine glands, has been reported [8]. Although we did not observe this in our biopsy, this phenomenon suggests a role for the eccrine epithelium in the disease process, which can be helpful for pathological diagnosis.

In our case, the patient's medical history (initial inflammatory vulvitis unresponsive to topical steroids and oral antibiotics, followed by LS after a four-week delay), physical examination, and laboratory findings (macerated, erythematous inguinal folds and a positive $\mathrm{KOH}$ test for Candida) suggested Candida-induced LS. Negative cultures, the absence of leukocytosis, and no increase in CRP or ESR also suggested a fungal infection. Several reports regarding cutaneous candidiasis-induced hypersensitivity reactions further support the hypothesis that Candida infection was the triggering factor in our case [9, 10]. Because the patient was first treated at another clinic, we have limited information on the initial stage of the vulvar disease or initial tests. Furthermore, as the blood tests ordered in our clinic may have been affected by previous treatments, we cannot exclude the possibility of bacterial infection or drug reaction as the initiating factor. LS is an uncommon disease, and most of our knowledge about this disease is derived from case reports, which are prominent references with respect to aetiological factors and treatment options. We present this case to suggest a probable relationship between candidiasis and LS.

Disclosure. Financial support: none. Conflict of interest: none.

${ }^{1}$ Kirikkale University Faculty of
Medicine, Department of
Dermatology and Venerology,
Yenisehir District, Tahsin Duru
Avenue, No.14, 71450 Yahsihan,
Kirikkale, Turkey
${ }^{2}$ Department of Pathology,
Yenisehir District, Tahsin Duru
Avenue, No.14, 71450 Yahsihan,
Kirikkale, Turkey
<gunduzozgur@ windowslive.com>

Özgür GÜNDÜZ
Rebiye ÇAKARTAŞ $^{1}$
Serkan DEMIRKAN $^{1}$
Pınar ATASOY

1. Hofer T. Lichen striatus in adults or 'adult blaschkitis'? Dermatology 2003; 207: 89-92.

2. Müller CS, Schmaltz R, Vogt $T$, Pföhler $C$. Lichen striatus and blaschkitis: reappraisal of the concept of blaschkolinear dermatoses. Br J Dermatol 2011 ; 164: 257-62. 
3. Taieb A, El Youbi A, Grosshans E, Maleville J. Lichen striatus: a Blaschko linear acquired inflammatory skin eruption. J Am Acad Dermatol 1991; 15: 637-42.

4. Smith SB. 3rd, Smith JB, Ellis LE, Hogan DJ. Lichen striatus: simultaneous occurrence in two nonrelated siblings. Pediatr Dermatol 1997; 14: 43-5

5. Yaosaka $M$, Daisuke $S$, litoyo $M$, Shibaki $A$, Shimizu $H$. Lichen striatus affecting a mother and her son. J Am Acad Dermatol 2005; 53: 352-3

6. Racette AJ, Adams AD, Kessler SE. Simultaneous lichen striatus in siblings along the same Blaschko line. Pediatr Dermatol 2009;26: 50-4.

7. Happle R. Transposable elements and the lines of Blaschko: a new perspective. Dermatology 2002; 204: 4-7.

8. Wang L, Chen F, Liu Y, Gao T, Wang G. Lichen striatus with syringotropism and hyperplasia of eccrine gland cells: a rare phenomenon that should not be confused with syringotropic mycosis fungoides. J Cutan Pathol 2016; 43: 927-31.

9. Bernstein JA, Seidu L. Chronic vulvovaginal Candida hypersensitivity: an underrecognized and undertreated disorder by allergists. Allergy Rhinol (Providence) 2015; 6: 44-9.

10. Chirac A, Brzezinski $P$, Chiriac AE, Foia L, Pinteala T. Autosensitisation (autoeczematisation) reactions in a case of diaper dermatitis candidiasis. Niger Med J 2014; 55: 274-5.

doi:10.1684/ejd.2018.3315

\section{Eosinophilia and systemic symptoms with transient ageusia: a drug reaction caused by zonisamide}

Drug-induced taste disorders (DITDs) represent a side effect of drugs and usually occur without other symptoms. To date, no drug eruption has ever been reported in association with DITD. We report herein a case of zonisamide-induced drug reaction with eosinophilia and systemic symptoms (DRESS) associated with transient but severe ageusia.

A 50-year-old man with epilepsy had been treated with sodium valproate, phenytoin, phenobarbital, and diazepam for six years. Three months after the additional administration of zonisamide, he developed a skin rash. We suspected drug eruption and administered systemic prednisolone (PSL) at $15 \mathrm{mg} / \mathrm{d}$ for a week (Day 1). The rash exacerbated, with exudative erythema covering $60 \%$ of the body surface, facial oedema, and lymphadenopathy, accompanied by fever $\left(39^{\circ} \mathrm{C}\right)$ (figure $\left.1 \mathrm{~A}\right)$. We clinically suspected DRESS and the patient was admitted to our hospital.

Blood tests revealed a leukocyte count of $28,300 / \mathrm{mm}^{3}$ (eosinophils: 17.5\%; lymphocytes: $32.5 \%$; neutrophils: 16.0\%; atypical lymphocytes: $14.5 \%$ ). Liver enzymes were increased, but renal function was normal. A skin biopsy from the rash showed a superficial perivascular dermatitis without individual cell keratinization or interface changes. The final diagnosis of DRESS was made by using the scoring system for DRESS [1] (table S1).

Zonisamide was changed to levetiracetam and increased the systemic PSL dose to $45 \mathrm{mg} / \mathrm{d}$. The rash improved and the dose of PSL was tapered (figure 1D). The patient had three peaks of fever (figure 1D). Human herpes virus 6 (HHV6) IgG titre was increased 10-fold (Day 7) to 2,560-fold (Day 22), suggesting HHV6 reactivation. At the first febrile peak,

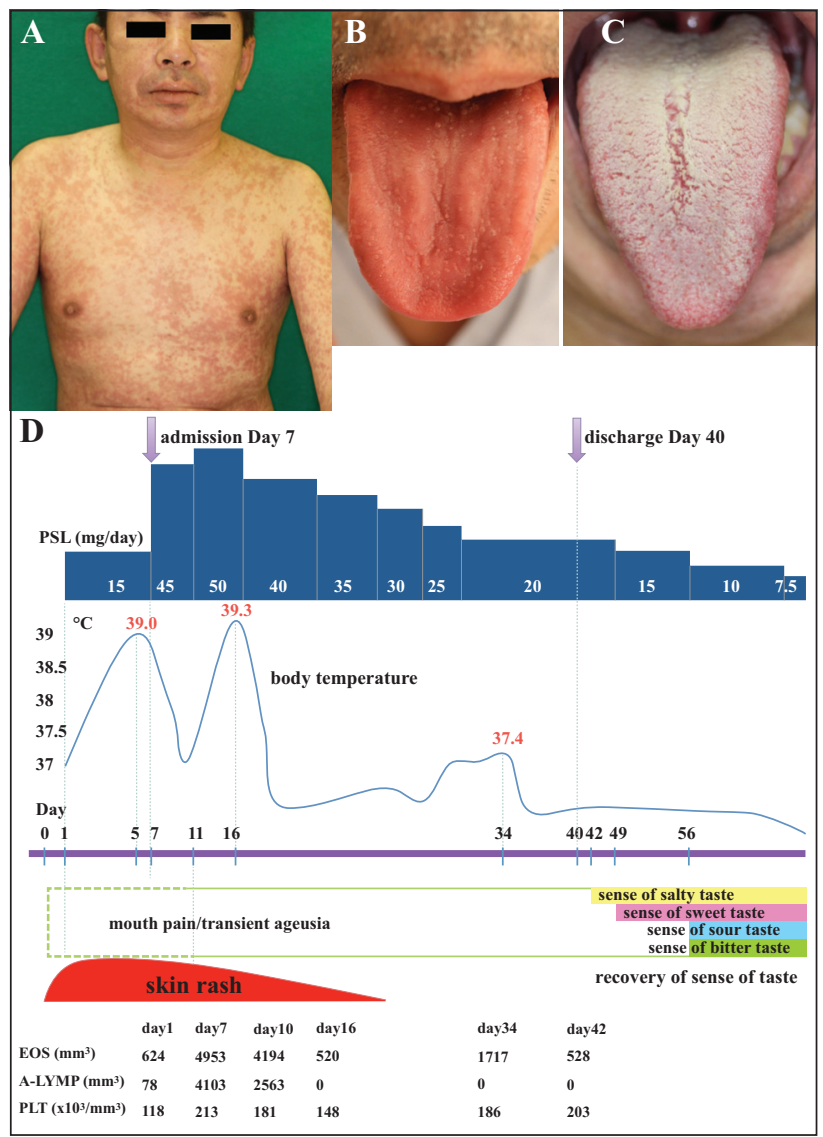

Figure 1. Clinical and pathological features, and clinical course of DRESS with ageusia caused by zonisamide. A) A skin rash is present on the whole body, including the face, upon admission (Day 7). B) The tongue is reddish and lacks a coating on Day 11, after the onset of DIHS. C) The tongue appears normal, with tongue coating apparent three months after the patient was discharged (the patient's sense of taste had recovered by this time). D) Clinical course of the patient. At the first febrile peak, the patient felt intraoral pain which hampered eating. After this peak, mouth pain was reduced, but a taste disorder developed. The patient was aware of ageusia on Day 11. The patient was followed in our outpatient clinic, and his ability to taste salty foods recovered on Day 42 , followed by his ability to taste sweetness, and then sour and bitter foods. Two months after the onset of DRESS, his ability to recognise all four types of taste had recovered. The absolute numbers of eosinophils (EOS), atypical lymphocytes (A-LYMP), and platelets (PLT) are shown chronologically.

the patient felt an intraoral burning sensation which was severe enough to hamper eating. He was only able to drink water. After the first febrile peak, mouth pain decreased, but he noticed ageusia. At that time, his tongue showed redness and a lack of coating (figure $1 B$ ). The Saxon test and wholemouth gustatory tests were performed by otolaryngologists, and brain magnetic resonance imaging (MRI) was carried out. All tests were negative except for the Saxon test with a result $(1.85 \mathrm{~g} / 2 \mathrm{~min}$; normal $>2 \mathrm{~g} / 2 \mathrm{~min})$ indicating a slightly dry mouth. Serum iron, zinc, and copper concentrations were within normal limits. Vitamin B12 level was 2,160 pg/mL (normal: $233-914 \mathrm{pg} / \mathrm{dL}$ ). The patient's ability to taste salt recovered 29 days after the onset of ageusia and nine days later, his ability to taste sweetness also recovered. 